\title{
Construcción y validación de un instrumento para medir conocimientos de madres y cuidadores sobre alimentación complementaria de infantes
}

\section{Construction and validation of an instrument to measure knowledge of mothers and caregivers on complementary feeding of infants}

\author{
Marco Sierra-Zúñiga ${ }^{a}$, Claudia Holguin Betancourt ${ }^{\mathrm{a}}$, Andry Yasmid Mera-Mamián ${ }^{\mathrm{b}}$, \\ Mario Delgado-Noguera ${ }^{a}$, José Andrés Calvache ${ }^{c}$
}

\author{
aDepartamento de Pediatría, Universidad del Cauca, Popayán, Colombia

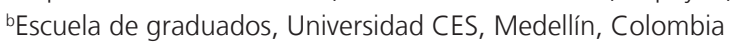 \\ 'Departamento de Anestesiología, Universidad del Cauca, Popayán, Colombia
}

Recibido: 29 de enero de 2019; Aceptado: 9 de julio de 2020

¿Qué se sabe del tema que trata este estudio?

La alimentación complementaria se constituye como un elemento clave en el desarrollo intelectual, físico y emocional de los infantes. Los conocimientos de madres y cuidadores son determinantes en las prácticas de alimentación de los niños bajo su cuidado.
¿Qué aporta este estudio a lo ya conocido?

Se presenta un instrumento rigurosamente construido y validado para medir conocimientos en alimentación complementaria a partir de lineamientos planteados por organizaciones líderes en la nutrición infantil, y con mejor calidad metodológica que las versiones disponibles en la región.

\section{Resumen}

La nutrición adecuada durante los primeros dos años de vida es fundamental para el desarrollo pleno del potencial del ser humano. La introducción inadecuada, precoz o tardía de la alimentación complementaria trae consecuencias a corto y largo plazo. La alimentación complementaria depende en gran medida de los conocimientos del cuidador que en los países de Latinoamérica generalmente es la madre. Objetivo: construir y validar un instrumento para medir los conocimientos maternos y de cuidadores sobre alimentación complementaria. Sujetos y Método: Estudio observacional. Participaron 80 madres comunitarias y 12 pediatras expertos. Se desarrolló en dos etapas: la creación del instrumento a partir de las 7 fases propuestas por Sampieri, y la validación mediante la evaluación de validez aparente, de constructo, de contenido, consistencia interna y fiabilidad intra-observador.
Palabras clave: Nutrición Infantil; Alimentación Complementaria; Niños;

Destete

Correspondencia:

Andry Yasmid Mera-Mamián

mera.andry@uces.edu.co 
Resultados: Se creó un instrumento autoadministrado que incluyó inicialmente 14 preguntas sobre conocimientos maternos y de cuidadores. Durante la validación de constructo se identificaron 3 dominios y se eliminaron cuatro preguntas. En la validación de contenido, 10 preguntas del instrumento definitivo obtuvieron calificaciones superiores a 9 (en una escala de 0-10) en las características: calidad, vocabulario, relevancia y actualidad. La consistencia interna global del instrumento fue moderada (Alfa de Cronbach: 0,64) y la fiabilidad intra-observador fue aceptable (k: 0,21-0,40) para el $80 \%$ de ítems del instrumento. Conclusiones: Se presenta el primer instrumento autoadministrado validado en la región, que permite medir conocimientos de las madres y los cuidadores sobre alimentación complementaria. A futuro, permitirá diseñar y desarrollar estrategias de evaluación e intervención en relación con los conocimientos maternos y de los cuidadores sobre alimentación complementaria.

\section{Abstract}

Adequate nutrition during the first two years of life is crucial for the full development of human potential. Inadequate, early, or late introduction of complementary feeding has consequences in the short- and long-term. Complementary feeding depends largely on the knowledge of the caregiver who, in Latin American countries, is usually the mother. Objective: To create and validate an instrument to measure knowledge about complementary feeding. Subjects and Method: Observational study in which 80 community mothers and 12 expert pediatricians participated. It was carried out in two stages, the creation of the instrument (following the 7 phases proposed by Sampieri) and the validation through the evaluation of the apparent validity, construct and content validity, internal consistency, and intra-observer reliability. Results: A self-administered instrument was created that initially included 14 questions about maternal and caregiver's knowledge. During the validation of the construct, 3 domains were identified and four questions were eliminated. In the content validation, 10 questions of the final instrument scored higher than 9 (on a scale of $0-10$ ) in the characteristics of quality, vocabulary, relevance, and topicality. The global internal consistency of the instrument was moderate (Cronbach's alpha: 0.64) and the intra-observer reliability was acceptable (k: 0.21-0.40) for $80 \%$ of its items. Conclusions: the first self-administered instrument validated in the region to measure the knowledge of mothers and caregivers about complementary feeding is presented. It will allow to design and develop strategies in relation to maternal and caregiver's knowledge of complementary feeding.

\section{Keywords:}

Child Nutrition;

Complementary

Feeding;

Child;

Weaning

\section{Introducción}

El periodo entre el nacimiento y los dos años de edad es una ventana de tiempo crítica para la promoción del crecimiento, la salud y el desarrollo óptimos ${ }^{1,2}$, siendo fundamental la nutrición para el desarrollo pleno del potencial de cada ser humano ${ }^{3}$. La Lactancia Natural Feliz es una recomendación global de salud pública, entendida como la práctica de la Lactancia Materna Exclusiva (LME), desde el nacimiento hasta el sexto mes de vida y continuando de allí en adelante con la introducción gradual de alimentos complementarios hasta los 24 meses en un proceso denominado Alimentación Complementaria (AC). Esta etapa de la vida infantil se considera importante dado que a partir del sexto mes de vida la LME ya no cubre las necesidades energéticas ni de micronutrientes del infante, por lo que es necesario ofrecer alimentos complementarios apropiados $^{4-7}$. Este proceso, considerado como un continuum, no debe ser entendido como un reemplazo, interrupción o interferencia con la lactancia materna $(\mathrm{LM})^{8}$.

Una adecuada introducción de la AC suplementa con micronutrientes como: vitaminas A, C y D, flúor, hierro, yodo y zinc, crea hábitos de alimentación adecuados. Esto estimula el desarrollo psicoemocional del niño porque es el momento del descubrimiento y percepción de sabores, colores, texturas, contacto con nuevos alimentos y objetos. De esta for$\mathrm{ma}$, se da inicio a una nueva etapa y por ende surgen necesidades adicionales en el proceso educativo con la familia .

La introducción precoz y tardía de la AC se ha asociado con consecuencias a corto y largo plazo. El inicio precoz de la AC se relaciona con un aumento significativo en la prevalencia de sobrepeso, obesidad ${ }^{10-13} y$ enfermedades respiratorias ${ }^{14}$. Además, podría generar daño renal debido a sobrecarga de solutos en un riñón inmaduro ${ }^{15} \mathrm{o}$ la aparición de anemia ${ }^{16}$. Por otra parte, el inicio tardío de la introducción de energía y nu- 
trientes, especialmente hierro y zinc, podría conducir a desnutrición ${ }^{16,17}$, lo que a largo plazo podría afectar el rendimiento intelectual, generar alteraciones generales de salud durante la adolescencia y la edad adulta, así como también producir problemas en desempeño laboral ${ }^{18}$.

En Colombia, la Encuesta Nacional de la Situación Nutricional en Colombia realizada en el 2010 (ENSIN 2010), resalta la magnitud del problema; pues reporta que la introducción de alimentos complementarios de la leche materna se realiza en forma precoz (1.8 meses), lo cual interfiere en la LME, además que la calidad de la dieta es especialmente deficitaria en niños y niñas de 6 a 8 meses, siendo poco común el consumo de frutas y verduras $^{19}$. La misma encuesta, realizada en 2015 (ENSIN 2015), mostró que la desnutrición aguda alcanzó un $2,3 \%$ y que el $41 \%$ de los niños con edades entre 6 y 23 meses tienen una dieta mínima aceptable, pero con frecuencia y variedad de alimentos deficientes ${ }^{20}$. Por su parte, el departamento del Cauca, en el suroccidente colombiano, es parte de las regiones con inseguridad alimentaria y nutricional del país ${ }^{21}$, presentando un retraso en el crecimiento infantil que supera el $20 \%$ y que clasifica esta prevalencia a nivel internacional como mediana ${ }^{19}$.

Además, de la adecuación de la AC en términos de tiempo, suficiencia, seguridad y adaptación, depende también de la disponibilidad de alimentos variados en el hogar y de los conocimientos y las prácticas de alimentación de la familia y los cuidadores, que generalmente en Latinoamérica y Colombia son las madres ${ }^{22}$. El grado de conocimientos y las prácticas maternas sobre AC, dependen de factores culturales y de recomendaciones realizadas por el personal de salud y personas cercanas $^{23}$. La falta de conocimiento básico nutricional y algunas creencias erradas llevan a que al menos el $50 \%$ de las madres tengan prácticas de alimentación inadecuadas .

En una revisión narrativa, realizada para la presente investigación, se encontró que en Latinoamérica las madres tienen conocimientos adecuados sobre LM, sin embargo, el conocimiento sobre AC fue insuficiente ${ }^{24}$. Este conocimiento, además, fue evaluado por instrumentos que fueron sometidos a procesos de validación incompletos y no contemplaban los dominios de la AC establecidos por la Organización Mundial de la Salud (OMS) ${ }^{1}$ y la Organización Panamericana de la Salud (OPS $)^{16}$. El objetivo del presente estudio fue construir y validar una herramienta para medir conocimientos maternos y de cuidadores de infantes sobre AC. Un instrumento de esta naturaleza, además de permitir una medición adecuada respecto del conocimiento de $\mathrm{AC}$, dará bases firmes para poder intervenir en aquellas poblaciones que lo requieran.

\section{Sujetos y Método}

\section{Tipo de estudio}

Estudio observacional en el cual se construyó y validó un instrumento autoadministrado. La etapa de construcción del instrumento se basó en las siete fases propuestas por Hernández Sampieri ${ }^{25,26}$ que son: redefiniciones fundamentales, revisión enfocada en la literatura, identificación de dominios y variables a medir, toma de decisiones clave, construcción, prueba piloto y generación de la versión final. En la etapa de validación de dicho instrumento, se midieron las propiedades psicométricas para valorar su validez y fiabilidad.

\section{Población y muestra}

Estuvo conformada por madres comunitarias, líderes técnicas en educación preescolar de los Jardines infantiles del Instituto Colombiano del Bienestar Familiar (ICBF), pertenecientes a la Red Interinstitucional y Comunitaria para la Promoción del Buen Trato en la infancia de Popayán-Cauca, Rico Buen Trato, que aceptaron su participación mediante la firma del consentimiento informado. El tamaño de la muestra de la población objetivo se definió en 80 participantes, es decir, 5 participantes por cada pregunta de conocimientos del instrumento, lo que está acorde con lo recomendado en la literatura ${ }^{27,28}$. Además, es importante señalar que previo a la aplicación del instrumento se hizo una prueba piloto con 40 madres comunitarias del ICBF, vinculadas a un jardín diferente a los que participaron en la etapa de validación propiamente. Finalmente, de las 80 madres que conformaron la muestra de validación, 57 fueron enroladas para la segunda medición, con la cual se evaluó las posibles variaciones intra-observador, lo que se conoce como "test-retest" por su denominación en idioma inglés.

\section{Recolección de la información, conducción del estudio y análisis estadístico}

\section{- Validez}

1. Validez aparente o lógica: Participaron 12 médicos especialistas en pediatría, de 32 a 64 años; experiencia profesional: 2 a 30 años; subespecialidad: 2 de ellos en cardiología pediátrica y cuidado intensivo pediátrico, de las ciudades colombianas de Popayán, Pasto y Cali, quienes por su cercanía con la Ciudad del grupo investigado poseen una mayor pertinencia lingüística respecto de la población objetivo. Además, se consultó a las 80 madres comunitarias, incluidas en el estudio, si consideraban que el instrumento permitía medir los conocimientos sobre AC, tal como se sugiere en la literatura $^{29,31}$. Esto se hizo mediante la pregunta: “ $i$ Usted considera que este instrumento permite medir conocimientos sobre alimentación complementaria?". 
2. Validez de contenido: Se consultó a los 12 médicos especialistas en pediatría (vía internet) si consideraban que los dominios y preguntas incluidas en el instrumento representaban de manera integral el concepto de $\mathrm{AC}^{29,31}$. El grupo de pediatras que participó en este proceso determinó para cada pregunta: 1) si el vocabulario era adecuado y 2) si la pregunta era clara, relevante y actual y asignó un puntaje en una escala de 0 a 10 . Se estableció que las preguntas que alcanzaron entre los evaluadores un promedio inferior a 6 serían revisadas por el grupo investigador. El grado de acuerdo entre los expertos se evaluó para cada ítem mediante el Coeficiente de Correlación Intraclase (CCI) considerando como malo o nulo (CCI $<0,20)$, mediocre (CCI $=0,21-0,40)$, moderado $(\mathrm{CCI}=0,41$ $0,60)$, bueno $(\mathrm{CCI}=0,61-0,80)$ o muy bueno $(\mathrm{CCI}=0,81-1,00)^{31-33}$.

3. Validez de criterio: Dado que este tipo de validez supone la existencia de un estándar de oro o Gold Standard ${ }^{29,34}$, no se realizó su valoración debido a la inexistencia de un estándar para la evaluación de los conocimientos en $\mathrm{AC}^{24}$.

4. Validez de constructo: Se evaluó mediante Análisis Factorial Exploratorio (AFE), el cual permite establecer si el constructo de $\mathrm{AC}$ es único o si al interior de este hay una estructura de dominios o factores $^{35}$. La evaluación del supuesto de colinealidad, el cual indica si el AFE es adecuado para la evaluación de validez de constructo, se hizo mediante la prueba Kaiser-Meyer-Olkin (KMO).

\section{- Fiabilidad}

1. Consistencia interna: Una vez identificados los factores, se evaluó la consistencia interna del instrumento mediante alfa de Cronbach ${ }^{36}$. Los resultados se presentaron para los ítems respecto de la puntuación global (correlación ítem-total) y para los ítems de cada dominio (correlación ítem-subescala), considerando aceptables aquellos valores de alfa de Cronbach $\geq 0,6$ y buena consistencia los valores $\geq 0,7^{29-31}$.

2. Fiabilidad intraobservador o estabilidad temporal: Se evaluó mediante la comparación de las respuestas registradas por 57 de las 80 participantes. El instrumento fue diligenciado por escrito en dos momentos diferentes, con quince días de diferencia. En ambas oportunidades fue llevado a cabo en un auditorio. Para su análisis se utilizó el coeficiente Kappa de Cohen ${ }^{29}$, el cual fue interpretado mediante la escala propuesta por Landis y Koch que clasificó la fuerza de la concordancia en seis niveles: pobre $(<0,01)$, leve $(0,01-0,20)$, aceptable $(0,21-0,40)$, moderada $(0,41-0,60)$, considerable $(0,61-0,80)$ y casi perfecta $(0,81-1)^{38}$. Dado que el instrumento fue una encuesta autoadministrado no se realizó evaluación de fiabilidad interobserva$\operatorname{dor}^{29}$.

Para todos los análisis se estableció un nivel de confianza del $95 \%$, un error alfa del $5 \%$ y un valor de $\mathrm{p}<0,05$. Los análisis se realizaron en SPSS 22.0 y $\mathrm{R}$ Statistics.

\section{Consideraciones éticas}

Según la resolución número 008430 de 1993, del Ministerio de Salud de Colombia el presente estudio fue considerado sin riesgo. Contó con la aprobación del Comité de ética del Hospital Universitario San José de la Ciudad de Popayán-Colombia (Acta de aprobación número 007 del 28 de julio de 2016).

\section{Resultados}

Los resultados del proceso de construcción del instrumento propuesto según las etapas de Hernández Sampieri se describen a continuación.

\section{Etapa I: Desarrollo y Construcción}

1. Fase I, redefiniciones fundamentales: Mediante reuniones periódicas del grupo investigador se revisaron los propósitos del estudio, la información que se deseaba recolectar con el instrumento y la población a quien iría dirigido.

2. Fase II, revisión enfocada en la literatura: Búsqueda estructurada: incluyó estudios originales y completos que evaluaron conocimientos maternos sobre AC en Latinoamérica ${ }^{24}$. Tiempo de búsqueda: enero de 2001 a junio de 2016. Bases de datos: MEDLINE (Pubmed), ScienDirect, EBSCO, LILACS, y Cochrane Library. Palabras claves en inglés: "nutrition, complementary feeding, child, weaning, knowledge". En español: "nutrición, alimentación complementaria, niños, destete, conocimientos, Latinoamérica”. Resultado de la búsqueda: 20 estudios, 12 de los cuales contaban con un instrumento de medición ${ }^{24}$.

3. Fase III, identificación de dominios y variables a medir: La revisión de estos estudios permitió iniciar la estructuración del instrumento, conformado por dos grandes dominios: 1) Variables sociodemográficas (edad, procedencia, estado civil, escolaridad, afiliación al sistema de salud colombiano, número de hijos, controles prenatales en el último embarazo, orientación recibida sobre LM y AC) y 2) Conocimientos en $\mathrm{AC}$ (definición, tiempo de inicio y finalización, frecuencia, tipo de alimentos, uso de suplementos nutricionales, consistencia, sistema y forma de suministro, lugar, cantidad y beneficios),según lineamientos de la $\mathrm{OMS}^{1,16}$. 
4. Fase IV, toma de decisiones clave: Se creó un instrumento con 16 preguntas sobre AC. Al revisar la última actualización realizada por la Sociedad Europea de Gastroenterología, Hepatología y Nutrición Pediátrica (ESPGHAN por sus siglas en inglés) sobre el tema, se eliminaron dos preguntas sobre el tipo de alimentos que deberían de iniciarse según la edad del lactante ${ }^{38}$.

5. Fase V, construcción: 24 preguntas: 10 sobre datos básicos y 14 sobre AC. El segundo grupo incluyó un enunciado inicial con una respuesta correcta y cuatro distractores de tipo binario (correcta versus incorrecta, o no sabe).

6. Fase VI, prueba piloto: Se aplicó a 40 participantes en un auditorio, en papel de forma autoadministrado. Tiempo promedio: 19 minutos (rango 1624). Adicionalmente, se consultó a las participantes, respecto a la sencillez, amenidad del formato, brevedad y claridad de las preguntas, identificando la necesidad de realizar algunas modificaciones (Anexo 1).

7. Fase VII, versión final: Se realizó una revisión de estilo por un experto externo, quién sugirió ajustes respecto al uso de palabras que permitieran la inclusión de ambos géneros; el uso de frases breves y claras; la organización en general de la forma del instrumento. Finalmente, el instrumento quedó conformado por: título, objetivo, instrucciones, preguntas, agradecimiento (Anexo 2).

\section{Etapa II: Validación}

En esta etapa del proceso, participaron un total de 80 madres comunitarias y 12 pediatras en el proceso de validación de contenido. El promedio de edad de las participantes fue de $43 \pm 12,5$ años (rango 19-74). La mediana del número de hijos fue 2 (rango 0-8). Las participantes reportaron una mediana de asistencia a controles prenatales en el último embarazo de 8 (rango 0-9). Otras características sociodemográficas se presentan en la tabla 1.

\section{Etapa IIA: Validez}

1. Validez aparente y de contenido: Todos los participantes manifestaron, que el instrumento creado permitía medir los conocimientos de madres y cuidadores sobre AC. El promedio de las calificaciones asignadas por los expertos pediatras a cada pregunta, respecto a las características de interés se mantuvo por encima de 9,0 para la mayoría de las variables, excepto respecto a la claridad de la pregunta sobre la definición de AC que obtuvo una calificación promedio de 8,3 (tabla 2). En ningún caso se obtuvieron puntajes promedios menores a 6.
Tabla 1. Características sociodemográficas de las madres participantes

\begin{tabular}{|c|c|c|}
\hline Variable & $\mathrm{N}$ & $\%$ \\
\hline \multicolumn{3}{|l|}{ Zona de procedencia } \\
\hline Rural & 68 & 85 \\
\hline Urbano & 12 & 15 \\
\hline \multicolumn{3}{|l|}{ Estado civil } \\
\hline Soltera & 27 & 33,8 \\
\hline Casada & 21 & 26,3 \\
\hline Unión libre & 24 & 30,0 \\
\hline Separada & 4 & 5,0 \\
\hline Viuda & 4 & 5,0 \\
\hline \multicolumn{3}{|l|}{ Escolaridad } \\
\hline Primaria & 1 & 1,3 \\
\hline Secundaria & 6 & 7,5 \\
\hline Técnica & 69 & 86,3 \\
\hline Universitaria & 4 & 5,0 \\
\hline \multicolumn{3}{|l|}{ Afiliación al sistema de salud } \\
\hline Contributivo & 73 & 91,3 \\
\hline Subsidiado & 6 & 7,5 \\
\hline Especial & 1 & 1,3 \\
\hline \multicolumn{3}{|c|}{ Orientación sobre lactancia materna } \\
\hline Sí & 62 & 77,5 \\
\hline No & 18 & 22,5 \\
\hline \multicolumn{3}{|c|}{ Persona que le brindó información sobre $A C$} \\
\hline Entidad de salud & 50 & 62,5 \\
\hline Familiar & 21 & 26,2 \\
\hline Ninguna de las anteriores & 9 & 11,2 \\
\hline
\end{tabular}

2. Validez de constructo: El AFE se inició con el análisis de la matriz de correlación entre todas las preguntas. Cuatro preguntas presentaron correlaciones lineales inferiores a 0.3 por lo cual fueron eliminadas. Estas preguntas fueron: 1) Número de veces al día que debe comer el niño(a) que inicia otros alimentos distintos a la leche, 2) Grupo de alimentos con los que se debe iniciar la alimentación complementaria, 3) Edad a la que el/la niño(a) puede ingerir alimentos que ingiere la familia, 4) Beneficios de la AC.

Con las 10 preguntas restantes se realizó nuevamente un AFE. Se encontró una regular correlación entre pares de variables $(\mathrm{KMO}=0,674)$ y una correlación lineal significativa entre las variables $\left(\chi^{2}=137\right.$; $\mathrm{p}<0,0001)$ lo cual dio paso a la realización del AFE. Se identificaron 4 factores intrínsecos. Uno de ellos fue suprimido por contener una sola pregunta y se forzó el análisis a extraer únicamente tres factores que explicaron el 54,8\% de la varianza total. Los resultados de los componentes rotados del AFE se presentan en la tabla 3. 


\begin{tabular}{|c|c|c|c|c|c|}
\hline & Preguntas & Vocabulario & Claridad & Relevancia & Actualidad \\
\hline 1 & Definición de alimentación complementaria & 9,0 & 8,3 & 9,5 & 9,0 \\
\hline 2 & Edad del niño(a) hasta la que se debe dar leche materna como único alimento & 9,7 & 9,7 & 9,7 & 9,6 \\
\hline 3 & Se debe suministrar suplementos nutricionales o multivitamínicos & 9,8 & 10,0 & 10,0 & 9,9 \\
\hline 4 & Consistencia de la comida del niño(a) según sus meses de edad & 9,5 & 9,2 & 9,9 & 9,2 \\
\hline 5 & Sistema adecuado para suministrar alimentos diferentes a la leche materna & 9,8 & 9,8 & 10,0 & 10,0 \\
\hline 6 & Lugar donde se deben dar los alimentos a el/la niño(a) & 9,8 & 9,8 & 10,0 & 9,8 \\
\hline 7 & Alimentos para evitar que el/la niño(a) tenga anemia & 9,8 & 9,9 & 10,0 & 9,9 \\
\hline 8 & Edad en que finaliza la alimentación complementaria & 9,7 & 9,8 & 9,8 & 9,7 \\
\hline 9 & Forma adecuada de dar alimentos complementarios & 9,8 & 9,6 & 9,9 & 9,4 \\
\hline 10 & Cantidad de alimentos complementarios que se deben suministrar a los 12 meses & 9,8 & 9,3 & 9,8 & 9,9 \\
\hline & Promedios totales & 9,7 & 9,5 & 9,8 & 9,6 \\
\hline
\end{tabular}

*Coeficiente de correlación intraclase (CCI) por categoría: Vocabulario = 0,568 IC95\% [0,031-0,873]; Claridad = 0,855 IC95\% [0,674-0,957]; Relevancia = 0,347 IC95\% [-0,466-0,808]; Actualidad = 0,588 IC95\% [0,74-0,879].

Tabla 3. Matriz rotada de los factores encontrados mediante Análisis Factorial Exploratorio y Alfa de Cronbach de cada factor

\begin{tabular}{lc}
\hline & \multicolumn{1}{c}{ Componente } \\
\hline Generalidades de la AC. Alfa de Cronbach $=0,64$ & 2 \\
Edad del niño(a) hasta la que se debe dar leche materna como único alimento & 0,732 \\
Consistencia de la comida del niño(a) según sus meses de edad & 0,715 \\
Sistema adecuado para suministrar alimentos diferentes a la leche materna & 0,624 \\
Se debe suministrar suplementos nutricionales o multivitamínicos & 0,611 \\
Tiempos para la administración de la AC. Alfa de Cronbach = 0,48 & 0,694 \\
Cantidad de alimentos complementarios que se deben suministrar a los 12 meses & 0,657 \\
Edad en que finaliza la alimentación complementaria & 0,654 \\
Definición de alimentación complementaria & 0,523 \\
Forma y lugar para la AC. Alfa de Cronbach = 0,51 & 0,470 \\
Forma adecuada de dar alimentos complementarios & 0,603 \\
Lugar donde se deben dar los alimentos a el/la niño(a) & 0,413 \\
Alimentos para evitar que el/la niño(a) tenga anemia & \\
\hline AC: alimentación complementaria & \\
\end{tabular}

\section{Etapa IIB: Fiabilidad}

1. Consistencia interna: El alfa de Cronbach global para todos los 10 ítems del instrumento fue de 0,60 , lo que indicó una fiabilidad moderada, fluctuando entre 0,48 y 0,64 entre los factores identificados por AFE (tabla 3 ).

2. Fiabilidad intraobservador: La aplicación realizada 15 días después de la primera vez ("test retest"), mostró una concordancia aceptable ( $\mathrm{k}$ : $0,21-0,40)$ para el $80 \%$ de ítems del instrumento $(\mathrm{p}<0,05)$. Los resultados se muestran en la tabla 4 .

\section{Discusión}

Esta investigación permitió la construcción del primer instrumento creado y validado en el Suroccidente colombiano, que mide conocimientos de las madres y cuidadores sobre AC adaptado al contexto de la región en la cual se trabajó. El proceso para su validación fue realizado con un alto rigor metodológico, acorde con las exigencias sobre métodos de validación.

Este estudio fue iniciado con una minuciosa revisión bibliográfica, la cual permitió establecer dominios 
Tabla 4. Kappa de Cohen en el análisis de concordancia "test retest"

\begin{tabular}{|c|c|c|c|c|}
\hline Pregunta & Índice de Kappa & Fuerza de la concordancia & Valor $\mathrm{p}$ & IC 95\% \\
\hline 1 & 0,3386 & $A$ & $0,01 *$ & $0,0808-0,5965$ \\
\hline 2 & 0,5371 & M & $<0,01$ * & $0,2603-0,8138$ \\
\hline 3 & 0,2899 & A & $<0,01$ * & $0,0377-0,5422$ \\
\hline 4 & 0,2400 & A & 0,33 & $-0,0142-0,4942$ \\
\hline 5 & 0,2845 & A & 0,015 & $-0,0707-0,6397$ \\
\hline 6 & 0,3049 & A & $0,01 *$ & $-0,1873-0,7971$ \\
\hline 7 & 0,4031 & A & 0,01 * & $0,1716-0,6347$ \\
\hline 8 & 0,3080 & A & $0,01 *$ & $0,1179-0,4982$ \\
\hline 9 & 0,1831 & L & 0,11 & $-0,0583-0,4245$ \\
\hline 10 & 0,3558 & $A$ & $<0,01$ * & $0,1793-0,5322$ \\
\hline
\end{tabular}

y variables para la construcción del instrumento, a la luz de las más recientes actualizaciones de la literatura mundial sobre $\mathrm{AC}^{1,16,38}$. Esto le brinda solidez en cuanto a la selección de los contenidos que fueron incorporados en la construcción de este instrumento.

Lo anterior podría explicar el hecho que la validación de contenido obtuviera una alta valoración. Es así como, el promedio de las calificaciones asignadas por los pediatras que oficiaron de expertos le otorgara a cada pregunta una evaluación por encima de 9, en la mayoría de las variables. Además, manifestando un grado de acuerdo de moderado a muy bueno, en los puntajes asignados a cada pregunta.

Por otra parte, la validez satisfactoria y las pruebas para la validez de constructo indican que el instrumento mide los conocimientos de madres y cuidadores sobre AC y, por lo tanto, se considera apto para ser empleado en futuras investigaciones.

El hecho que los resultados sean aceptables en la fiabilidad, medidos por la consistencia interna y la fiabilidad intra-observador, indican que el instrumento podría ser mejorado en el futuro. Sin embargo, es importante señalar que en estudios que evalúan conocimientos, se ha descrito que la evaluación de la confiabilidad intra-observador puede afectarse por el aprendizaje progresivo, sin que necesariamente medie algún tipo de capacitación en la temática de interés, lo cual pudo haber ocurrido en este estudio ${ }^{39,40}$.

En conjunto con lo anterior, cabe mencionar que, el resultado del AFE permitió eliminar preguntas que podrían resultar irrelevantes o que poco aportarían a la medición de AC. Esto mejoró la consistencia interna, pero podría haber afectado la validez de constructo del instrumento (reflejado en un 54,8\% de la varianza total explicada). Estas situaciones representan una posibilidad de investigación futura, en las cuales se podría explorar aspectos que pudieran mejorar la consistencia interna y la estructura de la herramienta ${ }^{41}$.

Aun cuando el instrumento generado con esta investigación podría ser perfeccionado, resulta un avance relevante el hecho de tener un instrumento que permita medir los conocimientos de los cuidadores sobre alimentación complementaria de manera objetiva. Esto hará que las investigaciones en este campo tengan resultados con mayor validez que las investigaciones actuales. En conjunto con lo anterior, la información recabada con este instrumento permitirá hacer intervenciones más cuidadosas a nivel de salud primaria, en esta etapa crucial de la alimentación infantil.

En otro orden, es importante destacar que, en Latinoamérica hay escasa literatura sobre el tema y los estudios primarios realizados en Perú y Ecuador, que abordan la evaluación de conocimientos maternos sobre AC en madres y/o cuidadores, presentan deficiencias en cuanto a la validación del instrumento empleado para la medición ${ }^{24}$. Además, los estudios realizados en población colombiana describen prácticas relacionadas con AC y conocimientos sobre LM, pero no se encontraron estudios que midieran los conocimientos sobre $\mathrm{AC}^{24}$. Esto podría deberse a la ausencia de un instrumento capaz e idóneo de realizar dicha medición.

Por otra parte, es importante considerar que la población latinoamericana en general y la colombiana, en lo particular, presenta una heterogeneidad cultural y geográfica, lo que conlleva a una variabilidad en lo que se refiere a alimentación. Es por lo que se recomienda ser cautos en la utilización de instrumento creado en este estudio sin una revisión previa y validación total o parcial si fuese necesaria, particularmente en regiones colombianas: Caribe, Andina, Amazónica, Orinoquía, Pacífica e Insular. 
Finalmente, el instrumento generado que tiene la posibilidad de constituirse en una herramienta apta como para medir el conocimiento de madres y cuidadores sobre AC. El uso de este cuestionario permitirá identificar a grupos de madres y cuidadores que necesiten apoyo en cuanto a AC, así como también conducir potenciales intervenciones de salud pública tendientes a mejorar el conocimiento sobre AC de la comunidad y los directamente involucrados en la alimentación de infantes.

\section{Conclusión}

El instrumento construido presenta una validez satisfactoria, una consistencia interna moderada y una fiabilidad aceptable. En ausencia de un instrumento similar, resulta adecuado para medir objetivamente los conocimientos de AC por parte de madres y cuidadores de infantes.

\section{Responsabilidades Éticas}

Protección de personas y animales: Los autores declaran que los procedimientos seguidos se conformaron a las normas éticas del comité de experimentación humana responsable y de acuerdo con la Asociación Médica Mundial y la Declaración de Helsinki.

Confidencialidad de los datos: Los autores declaran que han seguido los protocolos de su centro de trabajo sobre la publicación de datos de pacientes.

Derecho a la privacidad y consentimiento informado: Los autores han obtenido el consentimiento informado de los pacientes y/o sujetos referidos en el artículo. Este documento obra en poder del autor de correspondencia.

\section{Conflicto de intereses}

Los autores declaran no tener conflicto de intereses.

Anexo 1. Validación de un instrumento para medir conocimientos en AC. Cambios realizados en el instrumento posterior a la realización de la prueba piloto

- En la sección de identificación se debe retirar la pregunta número 1 que solicita escribir el nombre y la fecha de nacimiento, quedará solamente la opción que solicita la edad

- En la pregunta número 8, respecto al número de controles prenatales las encuestadas deben de colocar respuestas numéricas y en caso de no haber realizado controles se escribe el número cero

- La pregunta número 11, respecto a quién le brinda información sobre alimentación complementaria las opciones de respuesta serán más generales:

A. Un familiar

B. Por medio de alguna entidad de salud

C. Por algún medio tecnológico de información

- Respecto a la sección que evalúa conocimientos maternos, la pregunta número 15 , fue condensada de la siguiente manera: - ¿Con cuál grupo de alimentos se debe iniciar la alimentación complementaria? Elija una solo respuesta

- La pregunta número 19 que hace referencia a la inicio de suplementos multivitamínicos y nutricionales, la opción de respuesta C "Sólo a niños que indique el pediatra" debe cambiarse por la opción de respuesta "Sólo a los niños que indique el médico"

- La pregunta número 26 respecto a la forma adecuada de suministrar los alimentos complementarios, las opciones de respuesta fueron modificadas por problemas en la redacción de la misma quedando de la siguiente forma:

- Permitir que coma solo y experimente con diversas combinaciones y sabores

- Darle todos los alimentos de forma asistida ya que no están en capacidad de hacerlos por sí mismos

- Darle premios y juegos para que coma.

- No sabe 
Anexo 2. Validación de un instrumento para medir conocimientos en AC. Instrumento para la medición de conocimientos en alimentación complementaria

\section{INSTRUMENTO PARA LA EVALUACIÓN DE CONOCIMIENTOS MATERNOS O DEL CUIDADOR, SOBRE ALIMENTACIÓN COMPLEMENTARIA}

Marque con una $\mathbf{X}$ la respuesta que usted considere adecuada para las preguntas con opciones de respuesta multiple y complete la información en las preguntas que se continúan con una línea. Ingrese solo una opción para cada pregunta y por favor, no deje ninguna sin responder.

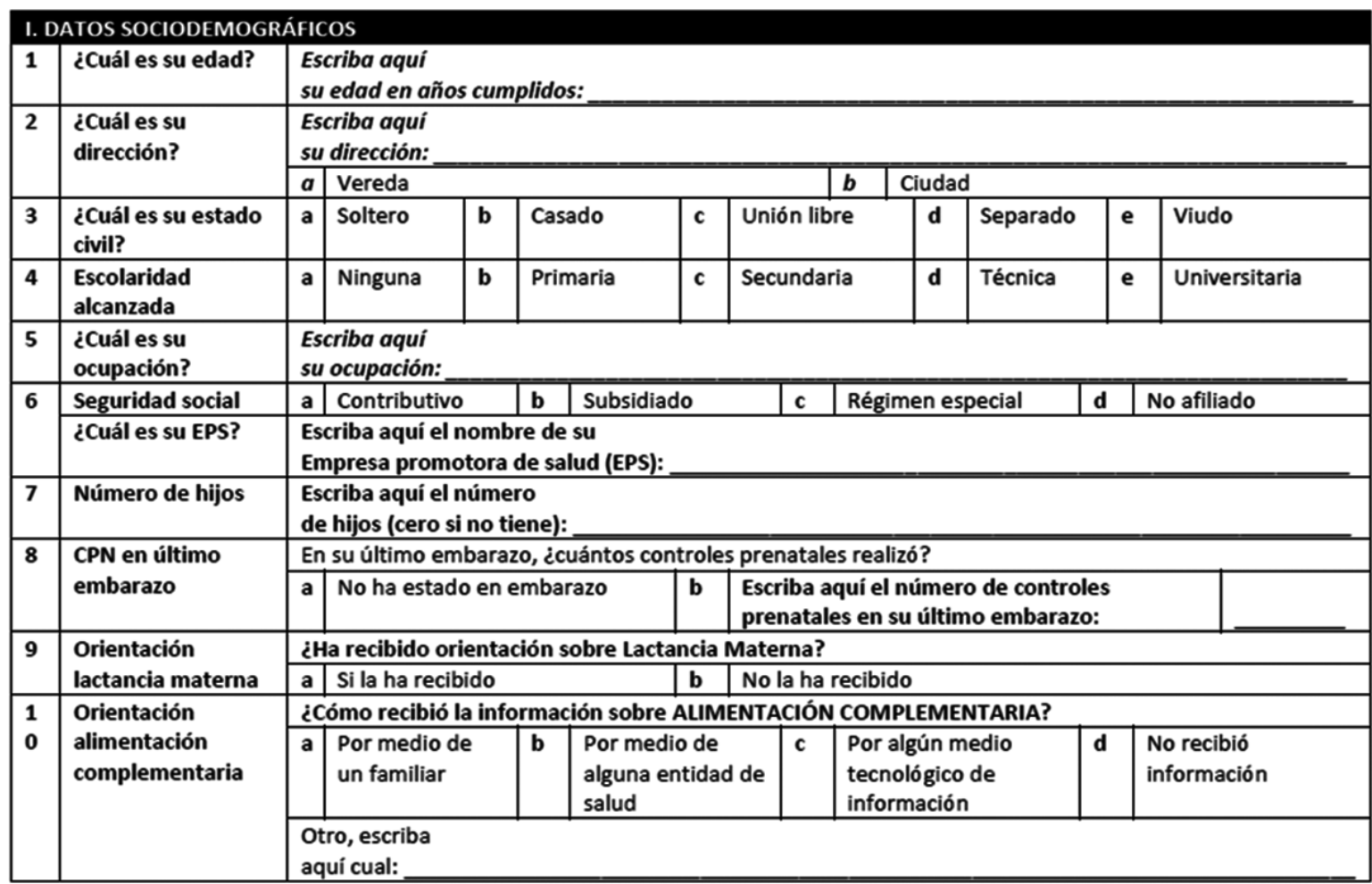

\section{CUESTIONARIO SOBRE ALIMENTACIÓN COMPLEMENTARIA}

INSTRUCCIONES: Marque con una $\mathrm{X}$ la respuesta que usted considere adecuada. Elija solo una opción para cada pregunta y por

favor no deje ninguna sin responder.

11. La alimentación complementaria es:

\begin{tabular}{|l|l|l|l|l|l|l|l|}
\hline a & Inicio de la dieta de la familia & b & $\begin{array}{l}\text { Inicio de alimentos sólidos y } \\
\text { líquidos diferentes a la leche } \\
\text { materna }\end{array}$ & c & $\begin{array}{l}\text { Inicio de alimentos } \\
\text { semisólidos solamente }\end{array}$ & No sabe \\
\hline
\end{tabular}

12. ¿Hasta qué edad del niño(a) se le debe dar leche materna como único alimento?

\begin{tabular}{|l|l|l|l|l|l|l|l|}
\hline a & Hasta los 6 meses & b & Hasta los 8 meses & c & Hasta los 2 años & d & No sabe \\
\hline
\end{tabular}

13. ¿Cuántas veces al día debe comer otros alimentos distintos a la leche el niño(a) que inicia la AC?

\begin{tabular}{|l|l|l|l|l|l|l|l|}
\hline a & $\mathbf{1}$ vez al día & b & $\mathbf{2}$ a 3 veces al día & c & 4 a 5 veces al día & d & No sabe \\
\hline
\end{tabular}

14. ¿Con qué grupo de alimentos se debe iniciar la alimentación complementaria?

\begin{tabular}{|l|l|l|l|l|l|l|l|}
\hline a & $\begin{array}{l}\text { Vegetales, cereales, frutas, } \\
\text { huevo y carne, progresando }\end{array}$ & b & $\begin{array}{l}\text { Vegetales, frutas y leche de } \\
\text { vaca }\end{array}$ & c & $\begin{array}{l}\text { Solamente frutas y } \\
\text { vegetales }\end{array}$ & No sabe \\
\hline
\end{tabular}


Anexo 2. Validación de un instrumento para medir conocimientos en AC. Instrumento para la medición de conocimientos en alimentación complementaria (continuación)

15. ¿A partir de qué edad el niño puede ingerir los alimentos que consume toda la familia?

\begin{tabular}{|l|l|l|l|l|l|l|l|}
\hline a & A partir de los 6 meses & b & A partir de los 8 meses & c & A partir de los 12 meses & d & No sabe \\
\hline
\end{tabular}

16. Los suplementos nutricionales o multivitamínicos se deben suministrar:

\begin{tabular}{|l|l|l|l|l|l|l|l|}
\hline a & $\begin{array}{l}\text { A los niños que luzcan delgados } \\
\text { y pálidos }\end{array}$ & b & $\begin{array}{l}\text { Solo a los niños que no } \\
\text { consumen leche materna }\end{array}$ & c & $\begin{array}{l}\text { Cuando lo indique el } \\
\text { personal de salud }\end{array}$ & d & No sabe \\
\hline
\end{tabular}

17. La consistencia de la comida del niño(a) según sus meses de edad, es:

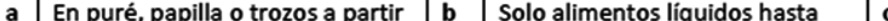
los 12 meses

En pequeños trozos a partir $\quad$ d de los 8 meses

18. La forma adecuada para suministrar los alimentos diferentes a la leche materna es:

\begin{tabular}{|l|l|l|l|l|l|l|l|}
\hline a & El tetero/biberón & b & Pitillo debido a la consistencia & c & $\begin{array}{l}\text { Cucharita, taza o dejar que } \\
\text { lo haga por si mismo }\end{array}$ & d & No sabe \\
\hline
\end{tabular}

19. El lugar donde se debe dar los alimentos al niño(a) es:

\begin{tabular}{|l|l|l|l|l|l|l|l|}
\hline a & En la sala & b & En el comedor & c & En el dormitorio & d & No sabe \\
\hline
\end{tabular}

20. Los alimentos que evitan que el niño (a) tenga anemia son:

\begin{tabular}{|l|l|l|l|l|l|l|l|}
\hline a & Leche y queso & b & Carnes y visceras & c & Caldos y coladas & d sabe \\
\hline
\end{tabular}

21. Si se le brinda una adecuada alimentación complementaria al niño(a), el beneficio será:

\begin{tabular}{|l|l|l|l|l|l|l|l|}
\hline a & $\begin{array}{l}\text { Que aumente de peso y luzca } \\
\text { rozagante }\end{array}$ & $\mathbf{b}$ & $\begin{array}{l}\text { Que tenga una ganancia de } \\
\text { peso y talla adecuados }\end{array}$ & c & $\begin{array}{l}\text { Que deje el seno de la } \\
\text { madre más temprano }\end{array}$ & d & No sabe \\
\hline
\end{tabular}

22. ¿A qué edad del niño(a) finaliza la alimentación complementaria?

\begin{tabular}{|l|l|l|l|l|l|l|l|}
\hline a & 12 meses & b & 18 meses & c & 24 meses & No sabe \\
\hline
\end{tabular}

23. La forma adecuada de dar alimentos complementarios es:

\begin{tabular}{|l|l|l|l|l|l|l|l|}
\hline a & $\begin{array}{l}\text { Permitir que coma solo y } \\
\text { experimente con diversas } \\
\text { combinaciones y sabores }\end{array}$ & b & $\begin{array}{l}\text { Darle todos los alimentos de } \\
\text { forma asistida }\end{array}$ & c & $\begin{array}{l}\text { Darle premios y juegos para } \\
\text { que coma }\end{array}$ & d & No sabe \\
\hline
\end{tabular}

24. La cantidad de alimentos complementarios que se deben suministrar a los 12 meses es:

\begin{tabular}{|l|l|l|l|l|l|l|l|}
\hline a & Hasta que esté totalmente lleno & b & $\begin{array}{l}\text { Media taza por comida }(\mathbf{1 2 5} \\
\mathrm{ml})\end{array}$ & $\mathbf{c}$ & $\begin{array}{l}\text { Una taza por comida (250 } \\
\mathrm{ml}\end{array}$ & d & No sabe \\
\hline
\end{tabular}

¡GRACIAS POR SU VALIOSO TIEMPO Y COLABORACIÓN!

\section{Referencias}

1. World Health Organization.

Complementary feeding: report of the global consultation, and summary of guiding principles for complementary feeding of the breastfed child [Internet]. 2003 [citado el 25 de agosto de 2019]. Disponible en: https://apps.who.int/iris/ handle/10665/42739.

2. Panjwani A, Heidkamp R.

Complementary Feeding Interventions
Have a Small but Significant Impact on Linear and Ponderal Growth of Children in Low- and Middle-Income Countries: A Systematic Review and Meta-Analysis. J Nutr. 2017;147(11):2169S-2178S.

3. Romero-Velarde E, Villalpando-Carrión $S$, Pérez-Lizaur AB, et al. Consenso para las prácticas de alimentación complementaria en lactantes sanos. Bol Med Hosp Infant Mex. 2016;73(5):33856.

4. Giugliani ER, Victoria CG.
Complementary feeding. J Pediatr (Rio J). 2000;76 Suppl 3(3): S253-62.

5. Fondo de las Naciones Unidas para la Infancia (UNICEF). Innocenti Declaration on the Protection, Promotion and Support of Breastfeeding 1990 - 2005 [Internet]. 2005. [citado 28 de diciembre de 2018]. Disponible en: https://www. unicef.org/nutrition/files/Innocenti plus15_BreastfeedingReport.pdf

6. Organización Mundial de la Salud (OMS)/Organización Panamericana de la 
Salud (OPS). La alimentación del lactante y del niño pequeño: Capítulo Modelo para libros de texto dirigidos a estudiantes de medicina y otras ciencias de la salud. Ginebra. 2010.

7. Daza W, Dadán S. Alimentación complementaria en el primer año de vida. CCAP. 2009;8(4):18-27.

8. Underwood BA, Hofvander Y. Appropriate timing for complementary feeding of the breast-fed infant. A review. Acta Paediatr Scand Suppl. 1982; 294:132.

9. Organización Panamericana de la Salud (OPS). ProPAN: Proceso para la Promoción de la Alimentación del Niño. Guía del Usuario del Programa de Cómputo Versión 2.0 [Internet]. 2013 [citado 25 de julio de 2018]. Disponible en: https://www.paho. org/hq/index.php?option=com_ docman\&view=download\&category slug=guia-usuario-programa-computo4989\&alias=22303-guia-usuario-esplores-303\&Itemid $=270 \&$ lang $=\mathrm{es}$

10. Aparicio Rodrigo M, García Vera CJ. La introducción precoz de la alimentación complementaria en los lactantes puede predisponer a obesidad. Evid Pediatr. 2011;7(41):2-4.

11. Popkin BM. Global nutrition dynamics: the world is shifting rapidly toward a diet linked with noncommunicable diseases. Am J Clin Nutr. 2006;84(2):289-98.

12. Wang J, Wu Y, Xiong G, et al. Introduction of complementary feeding before 4 months of age increases the risk of childhood overweight or obesity: A meta-analysis of prospective cohort studies. Nutr Res. 2016;36(8):759-70.

13. Grote V, Theurich M. Complementary feeding and obesity risk. Curr Opin Clin Nutr Metab Care. 2014;17(3):273-7.

14. Alzate-Meza MC, Arango C, CastañoCastrillón JJ, et al. A cross-sectional study of maternal breastfeeding as protection factor for prevalent diseases in children up to 5 years of age in some Colombian educational institutions, 2009. Rev Colomb Obstet Ginecol. 2011;62(1):5763.

15. Organización Mundial de la Salud (OMS), Organización Panamericana de la Salud (OPS), Instituto de Nutrición de Centro América y Panamá (INCAP). Alimentación infantil: Bases Fisiológicas. Editado por James Akre. 1992.

16. Ministerio de protección social. Atención Integrada de la Enfermedades Prevalente en la Primera Infancia AIEPI [Internet]. 2010 [citado 27 de septiembre de 2018]. Disponible en: https://www.minsalud.gov. co/sites/rid/Lists/BibliotecaDigital/RIDE/ DE/GUIA-PARA-LA-ATENCION-DELA-NINEZ-EN-LA-FAMILIA.pdf

17. Organización Mundial de la Salud
(OMS). Estrategia mundial para la alimentación del lactante y del niño pequeño. [Internet]. 2003 [citado 25 de julio de 2018]. Disponible en: https:// www.who.int/nutrition/publications/ gs_infant_feeding_text_spa.pdf

18. Agencia Española de Seguridad Alimentaria. Estrategia para la nutrición, actividad física y prevención de la obesidad [Internet]. 2005 [citado 1 de febrero de 2018]. Disponible en: http://www.aecosan.msssi.gob.es/ AECOSAN/docs/documentos/nutricion/ estrategianaos.pdf

19. Ministerio de Salud y Protección Social. Instituto Colombiano de Bienestar Familiar (ICBF). Encuesta Nacional de Situación Nutricional ENSIN [Internet]. 2010 [citado 1 de febrero de 2018]. Disponible en: https://www.javeriana. edu.co/documents/245769/3025871/ Resumen_Ejecutivo_ENSIN_2010. pdf/160e9856-006d-4a609da3-d71606703609.

20. Ministerio de Salud y Protección Social. Instituto Colombiano de Bienestar Familiar (ICBF). Encuesta Nacional de Situación Nutricional ENSIN [Internet]. 2015 [citado 2 de marzo de 2018]. Disponible en: https://www.icbf.gov.co/ bienestar/nutricion/encuesta-nacionalsituacion-nutricional

21. Instituto Colombiano de Bienestar Familiar (ICBF). Informe Planes Departamentales de Seguridad Alimentaria y Nutricional 2007-2008. 2009 [Internet]. [citado 15 de marzo de 2018]. Disponible en: http://bvsper.paho. org/texcom/nutricion/PLANESODEPART. pdf

22. Ministerio de la Protección Social de Colombia. Plan Nacional de Seguridad Alimentaria y Nutricional (PNSAN) 2012 -2019. 2012 [Internet]. [citado 30 de noviembre de 2017]. Disponible en: https://www.icbf.gov.co/sites/default/files/ pnsan.pdf

23. Unsihuay-Ureta FS. Conocimiento materno sobre alimentación complementaria y estado nutricional en el lactante menor. Comunidad Santa Isabel-Huancayo, año 2009. In Crescendo. 2011;2(1):147-160

24. Sierra Zúñiga MF, Holguín C, MeraMamián A, Delgado-Noguera M. Conocimientos maternos sobre alimentación complementaria en Latinoamérica: revisión narrativa. Rev Fac Cienc Salud Univ Cauca. 2017;19(2):2028.

25. Hernández Sampieri R, Fernández Collado C, Baptista Lucio P. Metodología de la investigación. 5.a ed. México D.F.: Mc Graw Hill; 2010.

26. Segredo Pérez AM, Pérez Piñedo J, López P. Construcción y validación de un instrumento para evaluar el clima organizacional en el ámbito de la salud pública. Revista Cubana de Salud Pública. 2015;41(4):603-19.

27. Hogan T. Pruebas psicológicas. Una introducción práctica. Manual Moderno México. 1a Edición. 2004.

28. Medrano LA, Trógolo M. Validación de la Escala de Dificultades en la Regulación Emocional en la población universitaria de Córdoba, Argentina. Univ Psy. 2014;13(4):1345-56.

29. Ramada-Rodilla JM, Serra-Pujadas C, Delclós-Clanchet GL. Adaptación cultural y validación de cuestionarios de salud: revisión y recomendaciones metodológicas. Salud Pública México. 2013;55(1):57-66.

30. Mateus-Galeano EM, CéspedesCuevas VM. Validez y confiabilidad del instrumento «Medición de la autoeficacia percibida en apnea del sueño» SEMSA. Versión en español. Aquichan. 2016;16(1):67-82.

31. Carvajal A, Centeno C, Watson R, Martínez M, Rubiales AS. ¿ How is an instrument for measuring health to be validated?. An Sist Sanit Navar. 2011;34(1):63-72.

32. Müller R, Büttner P. A critical discussion of intraclass correlation coefficients. Stat Med. 1994;13(23-24):2465-76.

33. Bland JM, Altman DG. Measurement error and correlation coefficients. BMJ. 1996;313(7048):41-2.

34. Versi E. «Gold standard» is an appropriate term. BMJ. 1992;305(6846):187.

35. Kang $\mathrm{H}$. A guide on the use of factor analysis in the assessment of construct validity. J Korean Acad Nurs. 2013;43(5):587-94.

36. Tan Ş. Misuses of KR-20 and Cronbach's Alpha Reliability Coefficients. Eğitim ve Bilim. 2009;34(152):101-12.

37. Landis JR, Koch GG. The measurement of observer agreement for categorical data. Biometrics. 1977;33(1):159-74.

38. Fewtrell M, Bronsky J, Campoy C, et al. Complementary feeding: A position paper by the European Society for Paediatric Gastroenterology, Hepatology, and Nutrition (ESPGHAN) committee on nutrition. J Pediatr Gastroenterol Nutr. 2017;64(1):119-32.

39. Luján-Tangarife, A J, Cardona-Arias, A J. Construcción y validación de escalas de medición en salud: revisión de propiedades psicométricas. Arch Med. 2015;11(3):1-10.

40. Armigón Pallás JM, Villa JJ. Métodos de investigación clínica y epidemiológica. Vol. 4ta. Barcelona, España: Elsevier; 2013.

41. Morrow JR, Jackson AW. How significant is your reliability?. Res Q Exerc Sport. 1993; 64:352-5. 